\title{
Massimo Stella
}

\section{Achille tra le rose (negli anni di Napoleone). Femminilizzazione del guerriero nella Penthesilea di Heinrich von Kleist ${ }^{1}$}

La tua destinazione, cara amica, o la destinazione della donna in genere, è indubbia e evidente; infatti, quale può essere se non quella di diventare madre e di educare alla terra uomini virtuosi? Buon per voi che la vostra destinazione sia cosi semplice e limitata! Per il vostro tramite la natura soltanto vuole raggiungere $i$ suoi scopi, mentre per mezzo di noi maschi anche lo Stato vuole raggiungere $i$ suoi, provocando spesso le più infauste contraddizioni. ${ }^{2}$

Heinrich von Kleist, Sull'educazione della donna, 16 settembre 1800

Geh, mein süßes Kind! Mars ruft dich! Du wirst den Pelëden dir bekränzen: werd'eine Mutter, stolz und froh, wie ich Heinrich von Kleist, Penthesilea, vv. 2137-2139

1 Dove non è altrimenti indicato le traduzioni sono mie.

2 La traduzione è di Marina Bistolfi, cfr. Heinrich von Kleist, Opere, a cura di Anna Maria Carpi, note di commento a cura di Stefania Sbarra, Milano, Mondadori, 2011. Quanto a Pentesilea, oltre all'edizione Mondadori per la collana I Meridiani testé citata, il lettore italiano può riferirsi all'edizione Marsilio con testo a fronte e traduzione di Paola Capriolo, cfr. Heinrich von Kleist, Pentesilea, tr. it. di Paola Capriolo, intr. di Rossana Rossanda, comm. di Claudia Vitale, Venezia, Marsilio, 2008. Traduzione memorabile quella di Ervinio Pocar, cfr. Heinrich von Kleist, Teatro, Parma, Guanda, 1992. 
Storicità di Penthesilea, Iliade del desiderio: dall'uomo fatale alla donna fatale

Penthesilea di Heinrich von Kleist è l'Iliade del desiderio. Che la guerra sia il significante iconico del desiderio è immemoriale topos culturale e poetico. Ma, nel momento in cui Kleist concepisce (tra il 1805 e il 1806) e pubblica (nel 1808) il dramma, la guerra assurge filosoficamente allo statuto di Idee. Il 13 ottobre del 1806 Napoleone entra a Jena. Celeberrime sono le parole con cui Hegel, in tempo reale, ne immortala l'immagine nella lettera a Niethammer:

Ho visto l'Imperatore, quest'anima del mondo, uscire dalla città per andare in ricognizione. $\grave{E}$ una sensazione meravigliosa vedere un tale individuo che qui, concentrato in un punto, seduto su un cavallo, si irradia sul mondo e lo domina. ${ }^{3}$

Al filosofo, che sta ultimando la Fenomenologia dello spirito, il guerriero e stratega Napoleone, Weltseele, "anima del mondo", sembra incarnare der Geist der Zeit, "lo spirito del tempo". ${ }^{4} \mathrm{E}$ la prospettiva si riconfermerà nelle Lezioni sulla filosofia della storia, dove Napoleone (con Alessandro e Cesare) è consacrato come «individuo cosmicostorico».

Ecco che cosa sono i grandi uomini nella storia: i loro scopi privati particolari racchiudono il contenuto sostanziale che è volontà dello spirito del mondo (Wille des Weltgeistes). Dobbiamo chiamarli eroi [...]. Perciò dobbiamo riconoscere agli uomini della storia mondiale (die welthistorischen Menschen), agli eroi di un'epoca, il merito di aver saputo capire; le loro azioni, i loro discorsi sono il meglio di quell'epoca [...]. Perciò gli altri seguono questi conduttori di anime (Seelenfiuhrern), poiché si sentono venire incontro la forza irresistibile del loro stesso spirito interiore. Se gettiamo uno sguardo sul destino di questi individui della storia mondiale (welthistorischen Individuen), i quali svolsero la professione d'incaricati d'affari dello spirito del mondo (Geschäftsfiuhrer des Weltgeistes), dobbiamo dire che non fu affat-

3 Georg Wilhelm Friedrich Hegel, Epistolario, a cura di Paolo Manganaro (2 voll.), Guida, Napoli, 1983-88, I, 1785-1808, p. 119 (mio il corsivo).

4 Beffardamente Goethe, nemico di tutte le rivoluzioni, in Faust, I, pp. 575577: «Quel che chiamate spirito dei tempi/ è in sostanza lo spirito degli uomini/ nei quali i tempi si rispecchiano./ E questo è spesso così meschino!/ Al primo sguardo si scappa via:/ solo immondizia e vecchia roba inutile,/ o tutt'al più tragedie di duci e paladini/ infarcite di massime di vita/ che stanno bene in bocca ai burattini!» (tr. it. di Andrea Casalegno). 
to felice. Essi non giunsero alla quiete del godimento, tutta la loro vita fu lavoro e fatica, la loro intera natura fu quell'unica passione. Muoiono presto, come Alessandro, o sono ammazzati come Cesare, oppure sono deportati a Sant'Elena, come Napoleone. ${ }^{5}$

L’uomo fatale, l'uomo «cosmico-storico», è per antonomasia uomo di guerra, poiché questo è lo strumento con cui l'Idee muove gli stati e i popoli non solo all'affermazione di sé, ma soprattutto al cambiamento e al perfezionamento. La guerra è un'astuzia della ragione (List der Vernunft) dietro alla quale l'Idee agisce.

Ma se Hegel è eccellente spettatore - entusiasta e simpatetico- di quel teatro bellico e geopolitico che culmina nella celeberrima battaglia di Jena (14 ottobre 1806), dall'altra parte del proscenio Carl von Clausewitz vi compare come attore in prima persona, sul campo, sul palcoscenico, vinto e prigioniero di guerra. È, appunto, von Clausewitz a fornire la parola-chiave che nomina l'Idee della guerra ovvero la sua "forma": Zweikampf.

Non entreremo in alcuna delle farraginose definizioni pubblicistiche della guerra, ma ci atterremo piuttosto al suo proprio elemento, e cioè al duello. La guerra non è altro che un duello su vasta scala. Se volessimo concepire come un'unità l'innumerevole quantità di duelli che compongono una guerra, lo faremmo al meglio se ci rappresentassimo due contendenti. Ciascuno fa ricorso alla violenza fisica per costringere l'altro a sottomettersi alla sua volontà. Il suo primo obiettivo è quello di far cadere l'altro e di renderlo così incapace di ulteriore resistenza. Sicché la guerra è un atto di violenza per costringere l'altro a compiere la nostra volontà. ${ }^{6}$

Che la riduzione della guerra moderna alla forma del duello sia un arcaismo è cosa evidente: prospettiva regressiva, posizione

5 Georg Wilhelm Friedrich Hegel, Lezioni sulla filosofia della storia, tr. it. a cura di Giovanni Bonacina e Livio Sichirollo, Roma-Bari, Laterza, 2003, p. 28.

6 «Wir wollen hier nicht erst in eine schwerfällige publizistische Definition des Krieges hineinsteigen, sondern uns an das Element desselben halten, an den Zweikampf. Der Krieg ist nichts als ein erweiterter Zweikampf. Wollen wir uns die Unzahl der einzelnen Zweikämpfe, aus denen er besteht, als Einheit denken, so tun wir besser, uns zwei Ringende vorzustellen. Jeder sucht den anderen durch physische Gewalt zur Erfüllung seines Willens zu zwingen; sein nächster Zweck ist, den Gegner niederzuwerfen und dadurch zu jedem ferneren Widerstand unfähig zu machen. Der Krieg ist also ein Akt der Gewalt, um den Gegner zur Erfillung unseres Willens zu zwingens (corsivo dell'autore), Carl von Clausewitz, Vom Kriege, hrsg. Werner Hahlweg, I, 1, 1, Bonn, Dümmler Verlag, 1991. 
di un grande conservatore dell'Ancien Régime a fronte del trauma napoleonico, ${ }^{7} \mathrm{e}$ più in fondo ancora, a fronte della Rivoluzione francese che diffuse il nuovo corso del Volkskrieg, della guerre de peuple.

Duello, guerra di popolo e «individuo cosmico-storico» sono i tre segni epocali che circoscrivono il movimento drammaturgico di Penthesilea. "Vedi su questi campi/ gli eserciti dei Greci e delle Amazzoni/ combattersi come due lupi rabbiosi» ${ }^{6}{ }^{-}$così Odisseo ad Antiloco. Wie zwei erboste Wölfe sich umkämpfen: l'immagine del Zweikampf, del duello, è evocata immediatamente in apertura dell'azione drammatica. Ma, al v. 556 della scena IV, l'esercito delle Amazzoni è definito, con disprezzo, da Diomede, Kriegsgesindel, «plebaglia guerriera»: come fossimo in un teatro di guerra napoleonico, le colline circostanti il campo troiano sono ricoperte da masse di nemici in armi, da nugoli di Amazzoni. ${ }^{9}$ Un soldato dolope descrive le schiere amazzoniche ein Knäuel, ein verworrener, «un viluppo confuso», tale che das Chaos war, / Das erst', aus dem die Welt sprang, deutlicher, «il Caos/ da cui il mondo scaturì, era meno intricato». ${ }^{10} \mathrm{E}$ il duello tra i contendenti, per un verso, e la ressa caotica, dall'altro, diventano, rispettivamente, su due piani diversi ma complementari, Idea del desiderio: il duello, Idea del desiderio che lega e divide Pentesilea e Achille; la ressa, Idea del «desiderio di specie», Begierde des Geschlechts ovvero Geschlechtstrieb, ${ }^{11}$ l'impulso sessuale, perché, come sappiamo,

7 Mi sembra pertanto errato interpretare von Clausewitz con lenti hegeliane. Hegel e von Clausewitz vanno certamente letti insieme, ma come poli opposti del dibattito sulla guerra. Né mi persuade la teoria che Hegel e von Clausewitz condividano il metodo dialettico nell'analisi, ma poi divergano sul piano etico-politico, cfr. Yuri Cormier, War as Paradox. Clausewitz and Hegel on Fighting Doctrines and Ethics, Montreal, McGill's-Queen University Press, 2016. Tantomeno mi pare valida la posizione di René Girard. Girard ritiene che la concezione clausewitziana della guerra come duello su vasta scala comporti logicamente l'innesco di un'interazione simmetrica tra i belligeranti tesa ad avviare un movimento incontrollabile di montée aux extrêmes, e in questo Girard riconosce una profezia dei conflitti globali postmoderni, cfr. René Girard, Achever Clausewitz, Paris, Carnets Nord, 2007.

8 Penthesilea, vv. 3-5: «Du siehst auf diesen Feldern,/ Der Griechen und der Amazonen Heer,/ Wie zwei erboste Wölfe sich umkämpfen».

9 Penthesilea, v. 543.

10 Penthesilea, vv. 436-438.

11 Prendo in prestito queste due espressioni da Arthur Schopenhauer, specificamente, dal cap. 42 «Leben der Gattung», dei Supplementi al mondo come volontà e rappresentazione, cfr. Supplementi a Il mondo come volontà e rappresentazione, a cura di Giorgio Brianese, Supplementi al libro IV, cap. 42, «La vita della specie», Torino, Einaudi, 2013, pp. 651-659. Non posso qui approfondire i legami di senso tra Penthesilea e la riflessione schopenhaueriana sulla volontà e il desiderio - per quanto al drammaturgo sia preclusa la conoscenza del filosofo, l'affinità problematica e tematica della tragedia kleistiana con il pensiero di Schopenhauer è molto profonda. Leggere il 
le Amazzoni sono piombate nel campo troiano per fare prigionieri gli uomini con cui si riprodurranno durante la misteriosa «Festa delle rose». Il duello del desiderio spicca in primo piano e ne occupa la scena; la mischia della libido costituisce invece lo sfondo della rappresentazione. E l'uno e l'altra sono violenza: violenza di una volontà contro una volontà - il duello; violenza involontaria della pulsione - la mischia.

E l'«Individuo fatale»?

\section{Pentesilea a cavallo, non Bonaparte}

Ho compiuto l'estremo, quanto le forze umane possono fare, - ho tentato l'impossibile -/ ho messo in gioco tutto;/ il dado, il dado decisivo, ora è fermo, è fermo;/ devo rendermene conto - che ho perso. ${ }^{12}$

Ecco le parole della Weltseele. A pronunciarle, questa volta, non è un uomo, un maschio, un eroe, un generale, un re, un Führer. È una donna, un'eroina, una Führerin. Non Alessandro, non Cesare, non Napoleone. Ma Pentesilea. E il rovesciamento è duplice perché nella tradizione antica è Achille a uccidere Pentesilea, mentre nel Trauerspiel kleistiano è Pentesilea che uccide Achille. Non potrebbe esserci enfasi più forte. Perché, allora, è una donna fatale e non un uomo ad occupare il centro della scena? Perché il welthistorisches Individuum, la Weltseele che incarna lo spirito del tempo, è donna? $\mathrm{E}$ in che senso è donna la Persona in cui l'Epoca s'incorpora e s'avvera?

Nella scena II, forse la più iconica del dramma, l'autore immortala l'Amazzone in una visione che sembra contendere antifrasticamente con la celeberrima tela di David, Bonaparte franchissant le Grand Saint-Bernard. ${ }^{13}$ Nel tentativo di piombare su Achille, l'Amazzone si inerpica sul suo cavallo per un dirupo tanto incastellato e scabroso da essere quasi una lama di roccia. Ha esplorato tutte le crepe per trovare un passaggio, vede che non c'è modo di scalare il burrone,

conflitto tra Wille, Wunsch e Begierde che si agita in Pentesilea sullo sfondo dell'inchiesta schopenhaueriana intorno all'Unberousst umano è illuminante.

12 Penthesilea, IX, vv. 1303-1307: «Das Äußerste, das Menschenkräfte leisten,/ Hab' ich getan - Unmögliches versucht - / Mein Alles hab' ich an den Wurf gesetzt;/ Der Würfel, der entscheidet, liegt, er liegt:/ Begreifen muß ich's - und daß ich verlor».

13 Di cui, come è noto, David realizza cinque versioni tra il 1801 e il 1803. Qui mi riferisco soprattutto alla prima, conservata al Musée national du Château de Malmaison. 
ma, come inventando uno spazio che non c'è, incomincia ad ascendere instancabilmente finché giunge ad un blocco di granito su cui a stento potrebbe reggersi un camoscio e da lì, dove non c'è più via d'uscita, si getta come un fulmine a capofitto verso le pendici della rupe, in un rovinare di pietre frananti, come dovesse precipitarsi negli Inferi. ${ }^{14}$ È lei, sulla scena kleistiana, l' «anima del mondo» in bilico sull'Abgrund, figura del Sublime, della Profondità e della Vertigine. Ma che cos'è dunque il Mondo che in lei si concentra ed esprime? Pur determinata nel suo intento e intrepida oltre ogni immaginabile limite, Pentesilea non trasuda quella compiaciuta e tranquilla sicurezza che appare sul volto giovanile del Bonaparte di David: se tutto intorno a lui volteggia e s'agita, il Console non si scompone e, saldamente eretto sul suo cavallo, indica con il dito meta e comando. Pentesilea, invece, si muove con la burrasca che suscita: è la iena dal cieco furore - die Hyäne, die blind-wütende, ${ }^{15}$ così almeno appare ai Greci che ascoltano il racconto del capitano. È tutta guidata dal suo desiderio di raggiungere l'avversario. Si tratta soltanto di cieco furore, come dice il greco Antiloco? O è un'altra forma, un altro modo d'essere e stare nel mondo, che nulla ha a che spartire con il dominio e il potere?

\section{Il guasto di Achille: il materno e il dolore}

L'Achille kleistiano è donna: il moderno Achille di Kleist prende il nome di «Pentesilea», eroe en femme, l'héros-femme. È questa una metamorfosi che nulla condivide con l'antico? O è forse proprio l'antico, l'Achille omerico, che suggerisce a un Kleist inquieto, visionario, eccentrico lettore dell'Iliade, la chiave per la straniante rigenerazione dell'eroe al femminile?

Non vi sono "fonti" classiche di questo dramma altre che l'Iliade (e, come vedremo, le Baccanti). ${ }^{16}$ Proprio la filologia ci insegna che le notizie frammentarie della tradizione indiretta sull'Etiopide, così come i récits mitografici sull'Amazzone, non hanno certo ispirato all'autore l'idea creativa della sua tragedia. Né certo si può pensare che le

14 Penthesilea, vv. 300-329.

15 Penthesilea, v. 331.

16 Vale forse la pena di ricordare che le Baccanti sono anche oggetto di particolare attenzione da parte di Hölderlin, a cavallo tra i due secoli. Non solo Hölderlin traduce parte del prologo, ma vede nel mito della nascita di Dioniso il mitologema dell'atto poetico moderno. Sulla rifunzionalizzazione del dionisiaco nelle poetiche del primo idealismo, si veda Manfred Frank, Il dio a venire. Lezioni sulla Nuova Mitologia, tr. it. di Flavio Cuniberto, Torino, Einaudi, 1994, pp. 6-19. 
informazioni tratte dal preziosissimo Gründliches mythologisches Lexicon (1770) di Benjamin Hederich (letto e riletto anche da Goethe) abbiano fornito a Kleist alcuna ispirazione poetica. Per Kleist, come per noi oggi, Pentesilea non è più che un nome, una leggenda, un'ombra. L'unica donna che abbia combattuto in guerra contro Achille. La rivale femminile di Achille, l'ombra femminile di Achille, verrebbe da dire... Di fatto, se c'è una "fonte" della Penthesilea, questa è l'Iliade, sulla cui scena, tuttavia, nessuna donna imbraccia le armi e tantomeno alcuna amazzone compare. Ma che cosa, allora, dell'Iliade omerica poté rigerminare nella forma della Penthesilea kleistiana?

Ecco gli snodi strategici. La pubblicazione nel 1795 dei Prolegomena ad Homerum di Wolf aveva, come è noto, decostruito l'idea dell'unità autoriale e quindi la coesione stessa del genere epico. Ed è proprio in conseguenza di questa disgregazione che August Schlegel nelle Vorlesungen über dramatische Kunst und Literatur avanza e percorre la possibilità d'una rilettura tragica dell'Iliade: Achille è il personaggio-chiave dell'interpretatio schlegeliana sub specie tragoediae. Qui, ancora una volta per la porta del tragico, entra in scena l'Achille di Hölderlin - molto più significativo, almeno in vista della Penthesilea kleistiana (per quanto impossibile la dipendenza interstestuale), dell'Achille reinventato da Goethe nella sua Achilleide. ${ }^{17}$ L'Achille hölderliniano quale ci appare dai due frammenti di Über Achill $(1799)^{18}$ è l'uomo del futuro o, meglio, sarebbe l'ideale cui l'uomo del futuro potrebbe tendere se il tempo in cui vive il poeta moderno non fosse quello di un irredimibile degrado: Achille è l'uomo della bellezza, dell'intuizione, dell'istinto, della rapidità, della Vita... proprio per questo è destinato, tragicamente, a una morte prematura e fatale. Achille è l'incarnazione della poetische Idee per eccellenza, là dove il poetico, il politico e l'etico vengono a coincidere in una sola scintilla. Ma soprattutto, l'Achille di Hölderlin è iscritto nel cerchio del dolore e del materno. ${ }^{19} \dot{E}$ il figlio della natura, l'enfant gâté della Natura, precisamente: «enfant gâté der Natur», come è detto nel frammento 2 di Über Achill. ${ }^{20}$ Perché enfant gâté? La

17 Per l'Achille di Goethe, rimando alle osservazioni di Sotera Fornaro: Johann Wolfgang von Goethe, Achilleide, Roma, Salerno editrice, 1998, pp. 24-33.

18 Nell'Hyperion Achille e Patroclo si fanno doppio della coppia omoerotica Iperione-Alabanda.

19 Interessanti osservazioni, per quanto concise, sull'Achille hölderliniano come "eroe moderno", si trovano nel libro di Jürgen Link, Hölderlins Fluchtlinie Griecheland, Göttingen, Vandenhoeck \& Ruprecht, 2020, pp. 219 e 234.

20 Riporto qui il testo del secondo frammento di Über Achill (cfr. Friedrich Hölderlin, Theoretische Schriften, hrsg. Johann Kreuzer, Hamburg, Meiner Verlag, 1998, pp. 24-25): «Am meisten aber lieb' ich und bewundere den Dichter aller 
formula è ellittica, allusiva, e richiede riflessione. Achille è l'eroe che per troppo amore di madre è stato reso quasi immortale: immerso nello Stige, ma non completamente. Sicché alla madre Achille sempre ritorna onde piangere i suoi dolori, il primo dei quali è la condanna a morire anzitempo e fuori patria: è la scena della lirica Achill. La madre di Achille, la Natura ovvero Teti, è ognora in lutto: ognora in attesa che le muoia quel figlio al contempo fortissimo e fragilissimo. Il guasto di Achille è l'amore materno: più precisamente, la mortalità è il sigillo dell'amore materno.

In questo specifico punto l'Achille antico e i suoi lettori moderni d'eccezione si incontrano. Nell'Iliade Achille è legato indissolubilmente al tema del dolore. Gregory Nagy, ${ }^{21}$ sulla scorta di Leonard Palmer ${ }^{22}$ ci ha indicato che, anzi, il dolore è iscritto nel nome di

Dichter um des Achilles willen. Es ist einzig, mit welcher Liebe und welchem Geiste er diesen Karakter durchschaut und gehalten und gehoben hat. Nimm die alten Herrn Agamemnon und Ulysses und Nestor mit ihrer Weisheit und Thorheit, nimm den Lärmer Diomed, den blindtobenden Ajax, und halte sie gegen den genialischen, allgewaltigen, melancholischzärtlichen Göttersohn, den Achill, gegen dieses enfant gaté der Natur, und wie der Dichter ihn, den Jüngling voll Löwenkraft und Geist und Anmuth, in die Mitte gestellt hat zwischen Altklugheit und Rohheit und du wirst ein Wunder der Kunst in Achilles Karakter finden. Im schönsten Kontraste stehet der Jüngling mit Hector, dem edeln treuen frommen Manne, der so ganz aus Pflicht und feinem Gewissen Held ist, da der andre alles aus reicher schöner Natur ist. Sie sind sich eben so entgegengesetzt, als sie verwandt sind, und eben dadurch wird es um so tragischer, wenn Achill am Ende als Todtfeind des Hector auftritt. Der freundliche Patroklus gesellt sich lieblich zu Achill und schikt sich so recht zu dem Trozigen. Man siehet auch wohl, wie hoch Homer den Helden seines Herzens achtete. Man hat sich oft gewundert, warum Homer, der doch den Zorn des Achill besingen wolle, ihn fast gar nicht erscheinen lasse. Er wollte den Götterjüngling nicht profaniren in dem Getümmel vor Troja. Der Idealische durfte nicht alltäglich erscheinen. Und er kont' ihn wirklich nicht herrlicher und zärtlicher besingen, als dadurch, daß er ihn zurüktreten läßt (weil sich der Jüngling in seiner genialischen Natur vom rangstolzen Agamemnon, als ein Unendlicher unendlich belaidiget fühlt) so daß jeder Verlust der Griechen, von dem Tag an wo man den Einzigen im Heere vermißt, an seine Überlegenheit über die ganze prächtige Menge der Herren und Diener mahnt, und die seltenen Momente, wo der Dichter ihn vor uns erscheinen läßt, durch seine Abwesenheit nur desto mehr ins Licht gesezt werden. Diese sind dann auch mit wunderbarer Kraft gezeichnet und der Jüngling tritt wechselweise, klagend und rächend, unaussprechlichrührend, und dann wieder furchtbar so lange nacheinander auf, bis am Ende, nachdem sein Leiden und sein Grimm aufs höchste gestiegen sind, nach fürchterlichem Ausbruch das Gewitter austobt, und der Göttersohn, kurz vor seinem Tode, den er vorausweiß, sichmit allem, so gar mit dem alten Vater Priamus aussöhnt. Diese lezte Scene ist himmlisch nach allem, was vorhergegangen war».

21 Gregory Nagy, The Best of Achaeans. The Concept of the Hero in Archaic Greek Poetry, Baltimore, Johns Hopkins University Press, 1979, pp. 70-83.

22 Leonard Palmer, The Interpretation of Mycenaean Greek Texts, Oxford, Oxford University Press, 1963, p. 79. 
Achille: Achilleus è forma abbreviata di *Akhí-lāuos "colui il cui popolo di guerrieri (laos) è afflitto". Sia o no questa etimologia da accettare, è invece indubbio che l'arte aedica e la lexis epica giocano sull'affinità paronomastica tra achos, "afflizione", e Achi-(l)leus. In effetti, per un verso Achille porta dentro se stesso il dolore di una vita breve, e per l'altro egli è ognora causa di dolore egualmente per gli Achei e per i Troiani: la violenza incontenibile delle sue pulsioni ed emozioni affligge tanto l'eroe quanto gli amici e i nemici. Il furore di Achille per l'oltraggio di Briseide, la celeberrima menis, campeggia nel primo verso dell'Iliade come cespite di infiniti lutti per l'esercito greco, mentre l'achos, l'afflizione, per la morte dell'amato Patroclo porterà all'uccisione di Ettore, perdita disastrosa per i Troiani. Dai dolori di Achille, infine, dipendono l'arresto e il riavvio della guerra: le laceranti emozioni dell'eroe sono, infatti, il motore macronarrativo del poema. Il culmine dell'achos di Achille, è, come si sa, l'uccisione di Patroclo. La reazione di Achille alla perdita dell'amato è del tutto anticonvenzionale, soprattutto per un guerriero: $:^{23}$ una nube nera lo avvolge, si versa la cenere in testa, si rotola e sporca nella polvere, si strappa i capelli, urla terribilmente... Antiloco gli tiene le mani perché ha paura che Achille si sgozzi con la spada in un accesso di furore. ${ }^{24}$ Non vuole più mangiare. Si stende su Patroclo e non fa che piangerlo ${ }^{25}$ e se si stende su di lui è per vegliare che le mosche non entrino nelle sue piaghe e non ne sfigurino il corpo. ${ }^{26} \mathrm{Si}$ staccherà dall'amato solo quando la madre gli promette di sostituirlo in quella veglia e di tenere, lei, lontana da Patroclo la razza selvaggia delle mosche che divora la carne dei guerrieri morti. ${ }^{27} \mathrm{Ma}$, appunto, è proprio nel dialogo con la madre, accorsa alle urla strazianti del figlio dal fondo del mare, che il dolore di Achille rivela la sua Forma.

Madre mia, $[. .$.$] / che piacere può esserci per me, se mi è morto l'a-$ mico, il compagno,/ Patroclo, che mi era caro più di tutti gli altri,/ come me stesso? [...]/ Oh, fossi restata tu tra le altre immortali del mare/ e Peleo avesse sposato una donna mortale!/ Ora anche per te ci sarà infinito dolore/ quando questo tuo figlio sarà morto,/ al

23 Per un'analisi del compianto di Achille su Patroclo, si vedano Christos Tsagalis, Epic Grief: Personal Laments in Homer's Iliad, Berlin-New York, De Gruyter, 2004, pp. 143-151; William C. Clarke, Achilles and Patroclus in Love, «Hermes», 106, 1978, n. 3, pp. 381-396.

24 Iliade, XVIII, 22-35.

25 Iliade, XIX, vv. 4-5.

26 Iliade, XIX, vv. 23-27.

27 Iliade, XIX, vv. 29-33. 
quale non è concesso tornare a casa,/ perché il cuore non mi spinge a vivere/ e a restare tra gli uomini/ [...]. Teti allora piangendo disse: "Sei vicino alla morte, figlio mio, da come mi parli:/ subito dopo Ettore ti è preparato il destino." / E, pieno d'affanno, rispose Achille dal piede veloce:/ "Potessi morire anche adesso: non ho difeso l'amico in pericolo di morte./ Molto lontano dalla patria è morto,/ e io che ero la sua difesa gli sono mancato./ E dal momento che io non devo tornare in patria mai più/ e che non fui luce per Patroclo né per gli altri compagni,/ [...] siedo qui presso le navi, inutile peso della terra,/ io che in guerra non ho pari tra gli Achei chitoni di bronzo. ${ }^{28}$

Achille, che piange Patroclo come si piangerebbe un figlio, specchia nella madre l'essenza e l'origine del proprio guasto.

Ebbene: non accade che anche Pentesilea - nel cui nome, come in quello di Achille, è iscritto il dolore, penthos - sia rinchiusa nel cerchio della fatalità materna, la legge delle madri? E non è nel cerchio della fatalità materna che Pentesilea farà esplodere la rivolta del desiderio?

\section{Effeminare Achille: l'illusione dell'Oggetto}

La scena XV, collocata al centro dell'azione drammaturgica, ${ }^{29}$ è il magico e unico momento di sospensione, di stasi si può ben dire, nel vasto, convulso movimento in cui consiste la Penthesilea kleistiana. Sappiamo che quella sospensione è soltanto apparente e, soprattutto, è frutto di un'illusione, se non di un inganno: Pentesilea, disarcionata da Achille, è caduta incosciente ai suoi piedi, sicché, di fatto, ne è la prigioniera. Ma Achille, pregato da Protoe, si piega alla finzione: risvegliatasi la regina dal mancamento, l'eroe le fa credere d'essere lui suo prigioniero, d'essere lui alla sua mercé. L'illusione, l'inganno e la finzione consentono l'incontro tra i due duellanti, un incontro che sarebbe altrimenti impossibile. Illusione, inganno e finzione non sono però un semplice dispositivo drammatico, un artificio della narrazione drammaturgica, e piuttosto costituiscono il sintomo di un evento cruciale e profondo: la costruzione e il contagio dell' $O g$ getto. In che senso?

28 Iliade, XIX, vv. 79-104.

29 Ripartita in ventiquattro scene, proprio come l'Iliade è ripartita in ventiquattro canti. Si tratta di un elemento largamente osservato dalla critica. 
Pentesilea Ora vieni, dolce figlio della Nereide,/ vieni stenditi ai miei piedi - più vicino/ Su, coraggio! Non avrai forse paura di me?/ Non mi odi, vero, perché ho vinto?/ Parla! Temi colei che ti gettò nella polvere?/ Achille Come i fiori la luce del sole Pentesilea Bene, ben detto! E allora guardami come se fossi il tuo sole./ [...] Ti prego, Pelide, non credere/ che io abbia mai attentato alla tua vita./ E vero che mi piaceva colpirti con questo braccio,/ ma quando tu cadesti,/ questo petto invidiò la polvere che ti accoglieva. ${ }^{30}$

Pentesilea è in estasi. Come una vergine tragica in festa, quasi fosse la folle Cassandra euripidea delle Troiane che irrompe sulla scena cantando e danzando l'Imeneo, ${ }^{31}$ la regina delle Amazzoni ha incitato il coro a cantare l'inno nuziale. ${ }^{32}$ Crede, da un lato, di possedere finalmente l'oggetto del suo desiderio, della sua passione. Dall'altro, si sente giunta a un limite estremo: zum Tode war ich nie so reif als jetz: «non sono mai stata così matura per la morte». ${ }^{33} \mathrm{E}$ la regina delle Amazzoni ripeterà questa stessa frase appena consumato lo scempio del corpo di Achille: Ich bin so selig [...]. Ganz reif zum Tod'o Diana, fühl'ich mich. ${ }^{34}$ L'identificazione tra desiderio e morte, come vedremo, è strutturale in Penthesilea: il mitema amor-mors è certamente di immemoriale tradizione, ma esso viene intellettualizzato e riorganizzato da von Kleist intorno al problema posto all'Essere-del-Soggetto dall'epifania dell'Oggetto.

Pentesilea è tanto fuori di sé dalla gioia quanto dubbiosa. Ha sognato di essere sconfitta da Achille: «Che sogno terribile ho sognato!/ [...] Mi è parso che nella mischia impetuosa/ la lancia del Pelide mi colpisse/ [...] Ero prigioniera, e tra risa di scherno ero condotta alla sua tenda». ${ }^{35}$ Nondimeno vuole celebrare la «festa delle rose», il Rosenfest, che consacra gli accoppiamenti delle Amazzoni con i compagni vinti e conquistati con le armi in battaglia.

Achille è il trofeo di Pentesilea - almeno così lei crede- e si trova dunque ad essere l'uomo con cui ella sembra destinata a congiungersi. Ma la regina vergine, che del sesso nulla sa - nulla della festa le è noto, dice, tranne il nome $-{ }^{36}$ il sesso non sa figurarsi, non sa, non

30 Penthesilea, vv. 1749-1756.

31 Penthesilea, vv. 1631-1664. Per le Troiane euripidee rimando invece ai vv. 308-340.

32 Per il canto corale, Penthesilea, vv. 1735-1742.

33 Penthesilea, v. 1682.

34 Penthesilea, vv. 2864-2865.

35 Penthesilea, vv. 1555-1571.

36 Penthesilea, vv. 2075-2076: «Feste [...] von denem mir bekannt nichts, als der Name: Rosenfest». 
può pensarlo. Piuttosto, cerca di immaginarsi, di fingersi nella mente e davanti agli occhi, di dar forma, infine, a colui che ora appare in suo dominio. Ma quale forma gli dà?

Pentesilea Ebbene, anche tu non ti muoverai più di una giovane colomba/ cui una vergine abbia passato i lacci intorno al collo/. Perché i sentimenti di questo petto, ragazzo, sono come mani, e ti accarezzano. Gli avvolge intorno le ghirlande Achille Chi sei tu, donna prodigiosa? Pentesilea Ti ho detto di stare fermo! Lo scoprirai presto./ - Ecco, soltanto queste spire di rose/ intorno al tuo capo, al tuo collo - giù sulle braccia, sulle mani, sui piedi - e di nuovo su verso il capo - ecco è fatto./ [...] Gli pone ancora una ghirlanda sul capo e lo lascia andare. Adesso ho finito. Oh, guarda [a Protoe], ti prego/, come gli si addice il diffuso splendore delle rose!/ Come brilla il suo volto scuro di tempesta!/ Il giovane giorno, davvero, mia carissima amica,/ quando le Ore lo conducono dai monti/ con perle e diamanti sotto i suoi passi,/ ecco, non appare così tenero e mite./ - Parla: non ti sembra che i suoi occhi brillino?/ - Davvero! A vederlo così, vien da dubitare/ che sia lui./ Protoe Ghe vuoi dire? Pentesilea Il Pelide! - Parla: fosti tu ad abbattere il più grande dei figli di Priamo/ di fronte alle mura di Troia? Sei davvero tu, tu, che con queste mani/ gli trafiggesti il piede in fuga, e dietro al tuo carro/ lo trascinasti intorno alla città paterna?/ - Parla! Di’! Che ti turba? Che ti prende? Achille Sono io. Pentesilea guardatolo attentamente Dice che è lui. ${ }^{37}$

37 Penthesilea, vv. 1769-1801: «PENTHESILEA Nun denn, so wirst du dich/ Nicht mehr als eine junge Taube regen,/ Um deren Hals ein Mädchen Schlingen legt./Denn die Gefühle dieser Brust, o Jüngling,/ Wie Hände sind sie, und sie streicheln dich. Sie umschlingt ihn mit Kränzen ACHILLES. Wer bist du, wunderbares Weib? PENTHESILEA Gieb her./ Ich sagte still! Du wirst es schon erfahren./ - Hier diese leichte Rosenwindung nur/ Um deine Scheitel, deinen Nacken hin - / Zu deinen Armen, Händen, Füssen nieder -/ Und wieder auf zum Haupt - so ist's geschehn./ Sie setzt ihm noch einen Kranz auf die Scheitel und läßt ihn gehn [...]. Jetzt ist's geschehn. - O sieh, ich bitte dich,/ Wie der zerfloßne Rosenglanz ihm steht!/ Wie sein gewitterdunkles Antlitz schimmert!/ Der junge Tag, wahrhaftig, liebste Freundinn,/ Wenn ihn die Horen von den Bergen führen,/ Demanten Perlen unter seinen Tritten:/ Er sieht so weich und mild nicht drein, als er./ Sprich! Dünkt's dich nicht, als ob sein Auge glänzte?/ Fürwahr! Man mögte, wenn er so erscheint, fast zweifeln,/ Daß er es sei. PROTOE. Wer, meinst du? PENTHESILEA. Der Pelide! - / Sprich, wer den Größesten der Priamiden/ Vor Trojas Mauern fällte, warst das du? / Hast du ihm wirklich, du, mit diesen Händen/ Den flücht'gen Fuß durchkeilt, an deiner Achse/ Ihn häuptlings um die Vaterstadt geschleift?/ Sprich! Rede! Was bewegt dich so? Wes fehlt dir? ACHILLES Ich bin's./ PENTHESILEA nachdem sie ihn scharf angesehen Er sagt, er sei's». 
Il gioco è chiaro: Wer bist du? Man mögte fast zweifeln, daß er es sei. Hast du ihm wirklich, du? Er sagt, er sei... - Chi sei tu, donna prodigiosa? - chiede Achille a lei. E di rimando Pentesilea a domandargli: - Sei tu, sei proprio tu? - e a commentare: - Verrebbe da dubitare che sia lui... Dice che è lui. L'incontro tra i due duellanti mette in crisi l'Essere. E la crisi è tutta di Pentesilea. Se infatti Achille la interroga: - Chi sei tu donna prodigiosa? - è solo per rimarcare il proprio stupore di fronte a una donna che sappia agire e parlare come lei. Achille non ha dubbi su Pentesilea: du sollst den Gott der Erde mir gebären, «Tu devi partorirmi il dio della Terra!», ${ }^{38}$ le dirà quando il velo dell'inganno cade alla fine della scena XV. Pentesilea, invece, ha un dubbio attanagliante, che la getta autenticamente nell'angoscia: chi è, che cos'è ciò che ha di fronte? È davvero lui il Pelide, l'uccisore di Ettore, del quale ha tanto sentito raccontare? È davvero lui quel «Marte in persona» (Mars selbst), quel «Dio della guerra» (Kriegsgott), che l'ha «abbagliata» (Geblendet stand ich), quando lo vide per la prima volta, «come quando di notte un fulmine cade dinnanzi al viandante» (wie wenn zur Nachzeit, der Blitz vor einen Wandrer fällt)? ${ }^{39} \grave{\mathrm{E}}$ proprio lui «l'eterno pensiero, l'eterno sogno» (ewiger Danke, ew'ger Traum) di Pentesilea? Quello la cui immagine Pentesilea vedeva intessuta «in ogni maglia grande e larga» (in jeder Masche, weit und groß) della «rete del mondo ornata di figure» (Die ganze Welt lag wie ein augespanntes Musternetz)? ${ }^{40}$

L'incontro con l'Oggetto del desiderio è alienante. Pone innanzitutto, a un primo stadio, il problema dell'avere, del possedere: Pentesilea è ossessionata dall'idea di vincere, cioè di avere in mano propria l'Oggetto-Achille. Ł̇ per questo che lo incatena "con le rose", che gli intima: Ich sagte still! -, che gli ordina: Ich bin's, du junger Griegsgott, der du angehörst: «Sono io, giovane dio della guerra, colei a cui appartieni», e ribadisce: wenn man in Volk dich fragt, so nennst du mich «se qualcuna del popolo te lo domanda, fa" il mio nome»; ${ }^{41}$ è per questo che, appena lo scorge sul campo di battaglia per la prima volta, promette a se stessa: Dich zu gewinnen, oder umzukommen, «o vincerti o morire». ${ }^{42}$ E per averlo, quel mirabile Oggetto, se lo crea con le parole, ne costruisce una Wortvorstellung, una rappresentazione di

38 Penthesilea, v. 2230.

39 Penthesilea, vv. 2211-2214.

40 Penthesilea, vv. 2187-2189.

41 Penthesilea, vv. 1806-1807.

42 Penthesilea, v. 2221. 
parola: $:^{43}$ che altro è, infatti, quell'incoronare di rose il capo di Achille e quell'avvincere tutto il suo corpo dalla testa ai piedi con catene di rose se non una scène de langage? Tanto più che rose è anagramma di eros $^{44}$ - Kleist gioca altresì enigmisticamente con il sintagma Amors $P f e i l,{ }^{45}$ «la freccia di Amore», dove il genitivo Amors riproduce la paraetimologia Amor-Mors. Pentesilea si dipinge dunque un'immagine dell'Oggetto con la parola rose. Ricorderemo tutti che cosa accade ad Aschenbach quando vede Tadzio après coup, per la seconda volta, quando cioè la bellezza del misterioso fanciullo non manifesta più tanto il suo immediato effetto meravigliante, quanto piuttosto il suo riposto lato angosciante, se non meduseo: ebbene, Aschenbach, spaventato da quella divina apparizione, le attribuisce una figura, das Haupt des Eros, vom gelblichen Schmelze parischen Marmor, «La testa di Eros, nello splendore dorato del marmo di paro». ${ }^{46}$ L'Oggetto del desiderio si incarna nel frammento di statua greca (e frammento d'un corpo), splendido reperto antico feticisticamente adorato... Pentesilea nomina, invece, e proietta eros nella forma anagrammatica di rose, che è poi il simbolo del sesso muliebre nella sua purezza e nella sua pienezza: nell'intera drammaturgia la rosa significa, duplicemente, sia la verginità delle giovani Amazzoni sia la loro maturità per le nozze. L'Achille-delle-rose, se così si può dire, è allora oggettivato al femminile: l'Achille effeminato, l'Achille incorporato, per dirlo in altri termini, alla Donna:

43 Come è noto, l'espressione e il concetto sono freudiani. La rappresentazione di parola, Wortvorstellung, è in relazione con la rappresentazione di cosa, Objektvorstellung o Sachvorstellung ovvero ancora Dingvorstellung. La tensione tra rappresentazione di parola e rappresentazione di cosa è già tematizzata in Sigmund Freud, Zur Auffassung der Aphasien, Leipzig, F. Deuticke, 1891, tr. it. L'interpretazione delle afasie, a cura di Francesco Napolitano, Macerata, Quodlibet, 2010: qui Freud si riferisce alla rappresentazione di cosa con il termine Objektvorstellung. Il termine di Dingvorstellung appare invece nella Traumdeutung (1900), Opere di Sigmund Freud, L'interpretazione dei sogni, a cura di Renata Colorni, tr. it. di Elvio Fachinelli e Herma Tretti, III, Torino, Bollati-Boringhieri, 1973. Il termine Sachvorstellung compare prevalentemente in Das Unbewusste (1915), Opere di Sigmund Freud, L'inconscio, tr. it. di Renata Colorni, VIII, Torino, Bollati-Boringhieri, 1976.

44 E risuona altresì nel nome Ares.

45 Penthesilea, v. 1075; v. 1082.

46 Thomas Mann, La morte a Venezia/ Der Tod in Venedig, a cura di Elisabeth Galvan, Venezia, Marsilio, 2013, cap. III, p. 120: «das Haupt des Eros, vom gelblichen Schmelze parischen Marmor». Bisognerebbe poi riflettere sull'immagine della testa assolutizzata, per così dire, ovvero staccata dal corpo, come immagine medusea della castrazione. Rimando al proposito, al celeberrimo saggio di Sigmund Freud, La testa di Medusa (1922), in Opere di Sigmund Freud, a cura di Cesare Musatti, IX, Torino, Bollati-Boringhieri, 1977. 
Achille Donna incomprensibile, chi sei?/ Che nome devo darti, quando la mia stessa anima/ rapita si domanda chi sei? PENTESILEA Se te lo domanda, nominale questi lineamenti: questo è il nome con il quale tu mi pensi. ${ }^{47}$

Il nome è il corpo stesso: Achille appartiene a quel corpo di donna. Ma l'Oggetto non è per nulla un doppio confusivo, è, al contrario, un'alterità che interviene nell'Io e lo scalza da sé: Pentesilea non vuole assimilarsi e assimilare Achille. ${ }^{48}$ L'immagine dell'Oggetto, d'altra parte, è illusoria, lo sappiamo, come ne è illusorio il possesso. Tuttavia, l'Oggetto non cade, resiste al di là della rappresentazione linguistica e immaginaria. Pentesilea si scontra infatti inevitabilmente con la domanda: che cos'è l'Oggetto che ho o fingo di avere? Sicché il desiderio dell'avere scivola verso il desiderio di essere: l'Essere viene diviso dall'Oggetto e trasformato in movimento. Ma desiderio d'essere che cosa? L'ombra dell'Oggetto cade su Pentesilea: ${ }^{49}$ ombra irrappresentabile, impronta cava della Cosa-in-sé, della Cosa perduta.

\section{L'Ombra di Otrera. Dall'Oggetto alla Cosa: Trauerbewegung}

L'Achille-Oggetto, l'Achille effeminato così dilegua. Quella splendida, luminosa figura di ragazzo tenero e mite, incoronato, avvinto e fragrante di rose e di donna si dissolve: non che non fosse reale per colei che l'ha creato. Lo era, reale, nell'intenzione: Pentesilea non parla, infatti, la lingua della Rede, del discorso retorico e convenzionale (T.S. Eliot lo chiamerebbe the dialect of the tribe). ${ }^{50}$ Ella parla, piuttosto, la lingua della poetische Sprache in cui non si esprime il com-

47 Penthesilea, vv. 1809-1815: «ACHILLES O du [...]/ Unbegreifliche, wer bist du? / Wie nenn ich dich, wenn meine eigne Seele/ Sich, die entzückte, fragt, wem sie gehört? PENTHESILEA Wenn sie dich fragt, so nenne diese Züge,/ Das sei der Nam', in welchem du mich denkst».

48 Per contro, molta bibliografia degli ultimi anni fonda l'analisi della relazione Pentesilea-Achille sul filo dello scambio di genere. Rimando soltanto, per un'interessante discussione, a Elystan Griffiths, Gender, Laughter and the Desecretion of Enlightment: Kleist's Penthesilea as Hundekomödie, «The Modern Language Review», 104, 2009, n. 2, pp. 453-471, dove il lettore troverà altresì una stimolante rassegna delle posizioni assunte dalla critica tedesca e internazionale sull'amazzonico Frauenstaat.

49 «Der Schatten des Objekts fiel so auf das Ich», scrive Freud in Trauer und Melancholie (1917), Opere di Sigmund Freud, Lutto e melancolia, tr. it. di Renata Colorni, VIII, Torino, Bollati-Boringhieri, 1976, p. 108.

50 The four Quartets, Little Gidding. 
mon credo ovvero la condizione, come Kleist la chiama,${ }^{51}$ bensì la parole del Soggetto che spesso, se non sempre, con la langue del Mondo si trova a mal partito scontrandosi con l'inesprimibile. ${ }^{52}$ Non è forse Pentesilea, a detta di Achille, unbegreifliche? L'Achille effeminato era reale per Pentesilea, ma non effettuale o convenzionalmente effettuale per il Mondo: reale, ma incomprensibile, come colei che lo ha pensato. E quanto Pentesilea credesse in quel bellissimo oggetto reale e illusorio, onirico, ma non fittizio, lo comprendiamo dal finale: quando la regina delle Amazzoni alza il velo che copre il cadavere di Achille scempiato e sfigurato dai suoi stessi denti; quando vede le rose di sangue (blut'gen Rosen) impresse dai propri morsi, e le ghirlande di ferite (Kranz von Wunden) intorno al capo, e quei boccioli cadere per la festa dei vermi (Knospen... zum Fest für die Gewürme) esalando un odore di tomba fresca, ${ }^{53}$ non vuole sapere chi lo ha ucciso. È disposta a perdonare l'uccisione e l'uccisore: perdona se stessa dell'omicidio compiuto. Ciò che vuole sapere, invece, e di cui vuole fare vendetta, è chi ha straziato l'immagine di quel corpo, la meravigliosa Forma di quel suo Oggetto. ${ }^{54}$

Sappiamo come termina l'ultimo duello. Pentesilea accetta la sfida di Achille. Con un terribile seguito di cani, di elefanti, di carri falcati e tizzoni ardenti, feroce come una belva, assetata di strage, brandito il grande arco, ella scende in campo ad affrontare il nemico. La Regina delle Amazzoni è più spaventosa e sanguinaria della Guerra stessa personificata. ${ }^{55}$ Achille l'aveva chiamata alla sfida solo per soccombere volontariamente e consegnarsi a lei, acconsentendo quindi a seguirla nel suo regno. Ma gli inganni o le astuzie a nulla valgono con Pentesilea (Achille aveva infatti sì l'intenzione di darsi all'Amazzone come prigioniero e seguirla a Temiscira, ma «solo per compiacerla in ciò che vuole; per una luna o due, non di più», e se poi lei volesse, a sua volta, seguirlo, l'eroe sarebbe ben felice «di farla

51 Sulla graduale produzione di pensieri durante il discorso, in Opere di Sigmund Freud, Lutto e melancolia, p. 995.

52 Nel corso della Penthesilea la divaricazione tra Rede e lingua poetica è ricorrente: evoco qui soltanto, per brevità, un punto, là dove Pentesilea erompe, infuriata, in queste parole: «Ciò che gli [ad Achille] ho sussurrato ha colpito il suo orecchio/ con la sola musica del discorso? (hat sein Ohr/ mit der Musik der Rede bloß getroffen)», vv. 2388-2389. I Greci parlano esclusivamente la lingua della Rede. Pentesilea, invece, parla un idioletto che è figura della poetische Sprache. E proprio in questo senso incorre nel fatale lapsus Küsse-Bisse, "baci-morsi", che ha perfettamente senso nella poesia, ma non nella Rede.

53 Penthesilea, vv. 2907-2910.

54 Penthesilea, vv. 2922-2936.

55 Penthesilea, vv. 2613-2617. 
sedere sul trono dei suoi padri»). ${ }^{56}$ Quando l'eroe greco le sia avvicina pieno di speranze, armato di una sola lancia, trova una Pentesilea circondata dai mastini, efferata e terribile cacciatrice. Tenta di nascondersi tra i rami di un abete. Pentesilea gli scaglia una freccia nel collo. Lui cade, ancora vivo. E lei, prima ad avventarsi su di lui, gli aizza quindi contro tutta la muta delle cagne: gli toglie l'armatura e lo sbrana, a gara coi veltri. ${ }^{57}$ Achille muore come il Penteo delle Baccanti euripidee, che sono senza dubbio il sottotesto della parte conclusiva. ${ }^{58}$ Perché proprio le Baccanti? Perché nella e dalla drammaturgia euripidea von Kleist incontra e preleva l'idea e l'immagine di un femminile-materno assassino e divoratore: l'idea e l'immagine di una collettiva Madre-Orco che sbrana i suoi figli. A tale idea e immagine Pentesilea è ricondotta. Perché e come?

Nel corso della scena XV, nel cerchio della sua apparente e illusoria quiete, Pentesilea si fa narratrice e racconta ad Achille il mito delle origini dell'Amazonenstaat. Tra le pieghe di quel lungo e complesso mythos sta riposta la relazione che lega Pentesilea a sua madre Otrera. E si scopre qualcosa di irregolare e imprevisto. È stata la madre a fare il nome di Achille sul letto di morte e indicarlo alla figlia come trofeo di guerra e sposo. ${ }^{59}$ Questo è del tutto irrituale, secondo la legge delle Amazzoni che, infatti, non devono cercarsi la preda, ma incontrarla per caso (anche se è auspicabile che incontrino, appunto, la più nobile). Pentesilea non voleva separarsi dalla madre: né per diventare regina succedendole nel regno, né tantomeno per partire alla guerra e alla cattura del designato prigioniero. Le Amazzoni dovettero trascinarla a viva forza sul trono. ${ }^{60}$

Piansi a lungo,/ per un'intera luna di dolore/ sulla tomba della scomparsa/ senza neppure prendere la corona,/ che giaceva sul margine senza padrone,/ finché il grido ripetuto del popolo/ che assediava impaziente il palazzo/ pronto per la spedizione guerriera/ mi trascinò con violenza sul trono. Comparvi,/ facendo forza a me stessa e piena di malinconia,/ nel tempio di Marte, dove il grande arco/ tintinnante del regno delle Amazzoni mi fu conse-

56 Penthesilea, vv. 2474-2475.

57 Per tutta la scena, si veda Penthesilea, vv. 2626-2672.

58 Non è questo il luogo di ricordare al lettore i molti prestiti dal testo di Euripide: rimando ai commenti delle citate edizioni Carpi-Sbarra e Capriolo- Rossanda-Vitale. Altro sottotesto antico da considerare è il racconto sulla fine di Penteo narrato nel III libro delle Metamorfosi ovidiane.

59 Penthesilea, v. 2141.

60 Penthesilea, v. 2157. 
gnato:/ ed era come se mia madre mi aleggiasse intorno,/ quando l'afferrai, e nessun dovere mi parve più sacro/ che adempiere le sue ultime volontà./ Cosparsi i fiori più profumati/ sul suo sarcofago,/ con l'esercito delle Amazzoni mi misi in marcia/ verso la rocca dei Dardanidi - per compiacere non tanto Marte/ il grande dio della guerra che mi aveva chiamata all'impresa,/ quanto l'ombra di Otrera./ [...] Io l'amavo. ${ }^{61}$

«Per compiacere l'ombra di Otrera», der Otrere Schatten zu gefallen, afferma Pentesilea. Per compiere le ultime parole di quell'ombra: «Va' mia dolce bambina, Marte ti chiama!/ Tu incoronerai il Pelide; / diventa una madre orgogliosa e felice come me». ${ }^{62}$ La «madre orgogliosa e felice» che Pentesilea avrebbe dovuto essere... si sovverte nell'Agave mitica che smembra a mani nude il proprio figlio. Come si è potuto produrre quest'esito mostruoso? Com'è che quel mandato materno si è trasformato in una missione d'orrore e di morte?

«Io l'amavo», dice Pentesilea, della madre, Ich liebte sie... La madre è stata l'unica esperienza dell'amore -non Wunsch, non Begierde, non Lust, non Trieb, ma Liebe, Amore- che Pentesilea abbia conosciuto. E l'esperienza è perduta. La madre, l'amore, sono la Cosa perduta: la madre "esterna", Otrera, è diventata la madre "interna", la Cosa, il vuoto della Cosa. Sicché il dramma, Spiel, di Pentesilea è il lutto, Trauer: letteralmente Trauerspiel. ${ }^{63} \mathrm{E}$ il lutto la forza che muove il desiderio della fanciulla, il penthos, appunto, quel dolore e quella nostalgia della perdita che stanno iscritti nel suo stesso nome. Ed è proprio questo il punto in cui, come già dicevamo qualche pagina indietro, l'Achille antico dell'Iliade e la Pentesilea kleistiana si congiungono e si sovrappongono, all'insegna della loro vulnerabilità materna. Forse la stessa eterotopia antica e greca della Penthesilea è, nel suo complesso e in sé stessa, la forma poetica, narrativa e inventiva del compianto sul Verlust.

L'idealizzazione, il diniego, l'onnipotenza, l'ambivalenza, la maniacale pulsione a trionfare sull'oggetto, a controllarlo e umiliarlo, tutti questi tratti nettissimi e marcati di cui il personaggio che chiamiamo Pentesilea dà ampia prova nella drammaturgia si riconducono al 'movimento', Bewegung, del lutto. ${ }^{64}$ Il luogo dell'Oggetto-Achille,

61 Penthesilea, vv. 2150-2169.

62 Penthesilea, v. 2137-2139. Mio il corsivo.

63 Sul concetto di Trauerspiel rimando a Walter Benjamin, Ursprung des deutschen Trauerspiels (1928), tr. it. di Enrico Filippini, Il dramma barocco tedesco, a cura di Cesare Cases, Torino, Einaudi, 1980.

64 Per intendere il "movimento del lutto" non è solo né tanto a Freud cui dovremo riferirci, ma soprattutto alle fondamentali considerazioni di Melanie Klein, 
l'Achille-delle-rose, la posizione, per così dire, che quest'immaginedi-parola va ad occupare per sprofondarvi è la vuota impronta della Cosa: la nostalgia dell'Origine è infera. Psicologismo? Lettura metapsicologica della Pentesilea kleistiana?

\section{La rivolta (im)politica di Pentesilea}

Riflettiamo. Il mito del Mutterrecht -dello Staat e del Diritto materni ovvero matrifocali - che von Kleist tematizza nella Penthesilea ha una radice indubbiamente memoriale-politica. È la Rivoluzione francese a mettere in movimento la macchina mitologica e mitopoietica delle eterotopie (più che delle utopie) comunitarie. E l'immagine dello stato rivoluzionario democratico, come ha messo in luce Lynn Hunt, ${ }^{65}$ si fregia, soprattutto nella prima fase della Révolution, del volto femminile e materno: celeberrimo il revival ideologico del culto di Iside Madre ${ }^{66}$ Alla Nation des Amazones è dedicato un articolo dell'Encyclopédie; Anne-Josèphe Théroigne de Méricourt, la cosiddetta Amazon rouge, medita di reclutare una falange di Amazzoni, nel 1792, per contribuire alla guerra; nel 1790, le Dames citoyennes de Saint Martin offrono all'Assemblea Nazionale i loro servigi sous le tître de Legion des Amazones... e gli esempi, più o meno significativi, si potrebbero moltiplicare. Se nel 1861 comparirà Das Mutterrecht di Johann Jakob Bachofen, ciò avviene anche per effetto di quel risveglio rivoluzionario e dei suoi numerosi sintomi culturali, nonostante l'autore declini poi il mito in tutt'altra direzione. ${ }^{67}$ L'operazione kleistiana è dunque piuttosto chiara, almeno nella sua genealogia, se non nel senso ultimo: il suo è un recupero segnato dai traumi storici. E si tratta altrettanto certamente di un recupero antifrastico: Kleist aveva assistito alla trasformazione della Rivolu-

Mourning and its Relation to Maniac-Depressive States (1940), in Ead., The Writings of Melanie Klein, II, Love, Guilt and Separation and Other Works, pp. 344-69, tr. it. di A. Guglielmi, Il lutto e la sua connessione con gli stati maniaco-depressivi, in Melanie Klein, Scritti. 1921-1958, Torino, Boringhieri, 1978.

65 Lynn Hunt, The Family Romance in the French Revolution, Berkeley and Los Angeles, University of California Press, 1992.

66 Sul culto rivoluzionario di Iside, rimando a Jurgis Baltrušaitis, La ricerca di Iside. Saggio sulla leggenda di un mito, tr. it. di Anna Bassan Levi, Milano, Adelphi, 1985; si veda anche, al proposito, Madelyn Gutwirth, The Treilight of the Goddesses: Women and Representation in the French Revolutionary Era, New Brunswick, Rutgers University Press, 1992, pp. 252-284.

67 Sull'orientamento ideologico del Mutterrrecht bachofeniano rimando a Furio Jesi, Recessi infiniti del «Mutterrrechb», in Johann Jakob Bachofen, Il matriarcato, tr. it., a cura di Giulio Schiavoni, I, Torino, Einaudi, 1988, pp. XIII-XXV. 
zione in Impero. Jena era una ferita aperta. L'anomalo Staat delle Amazzoni è un oggetto ambivalente: ha sì un'origine nella rivolta, per un verso, ma per l'altro identifica Legge e Natura e Forza assoggettando completamente l'Individuo: com'è ambiguo il mandato di Otrera che invia la figlia Pentesilea in guerra per il bene dello Stato, per la riproduzione e la conservazione dello Stato! Kleist gioca il brivido dell'ambivalenza, aprendo il dubbio e la domanda, sullo sfondo memoriale delle campagne napoleoniche. ${ }^{68}$ Ed eclatante è d'altra parte, come gli studi storici delle donne hanno da più parti segnalato all'attenzione critica, la confusività ideologica su cui si regge l'identificazione mito-politica tra il Materno e la comunità di liberi e uguali.

Il tema del Materno nella Pentesilea non è dunque di ordine psicologico o metapsicologico, ma mito-politico o poietico-politico, se si preferisce. Tuttavia, il tempo di von Kleist è il tempo della Filosofia del Soggetto, kantiana e idealistica. ${ }^{69}$ Ed è appunto sul versante della problematizzazione trascendentale e idealistica del Soggetto che si apre nella Penthesilea kleistiana il discorso del desiderio, dell'alienazione, della crisi dell'essere, dell'Oggetto e del rapporto tra Cosa e Rappresentazione. $^{70}$

In questo spazio filosofico, pertanto, nonché all'incrocio con la questione politica, si muove il confronto di Pentesilea con la figura materna, con la Cosa e l'Origine, o con la Cosa originaria. E qui si compie altresì la rivolta dell'Amazzone. Per Pentesilea la Madre

68 Dirk Grathoff, Die Sprachen der Penthesilea, in Id., Kleist: Geschichte, Politik, Sprache. Aufsätze zu Leben und Werk Heinrich von Kleists, Wiesbaden, Westdeutscher Verlag, 2000, pp. 132-128. Nello stesso volume si veda anche il capitolo Heinrich von Kleist und Napoleon Bonaparte, der Furor Teutonicus und die Ferne Revolution, pp. 175-197, che mette a fuoco il rapporto Kleist-Bonaparte. Ringrazio Stefania Sbarra per la discussione intorno a questi punti così problematici del testo kleistiano.

69 Von Kleist (come, del resto, per altro verso, i poeti e gli intellettuali del cenacolo di Jena) vive la rivoluzione kantiana e trascendentale del soggetto. Non sappiamo quanto di Kant von Kleist avesse realmente letto e in che misura, ma ben nota è l'affermazione del drammaturgo-scrittore secondo cui fu l'incontro con la filosofia di Kant a sconvolgere la sua visione della realtà. Il trascendentalismo kantiano apre quindi direttamente la porta all'Idealismo e alla sua visione dialettica del Soggetto. Von Kleist vive a cavallo tra queste due esperienze. C'è poi l'onda lunga della riflessione roussoviana sulla soggettività che giunge in Germania nell'età di Goethe e tocca da vicino von Kleist. Su questo, rimando a Stefania Sbarra, $L a$ statua di Glauco. Letture di Rousseau nell'età di Goethe, Roma, Carocci, 2006, pp. 202-240.

70 Come è noto, nel Seminario VII (1959-1960) Lacan sottolinea che Kant «meglio di chiunque altro, ha intravisto la funzione di Das Ding, anche se l'ha affrontata seguendo soltanto le vie della filosofia della scienza», cfr. Jacques Lacan, Il Seminario. Libro VII. L'etica della psicoanalisi, tr. it. di Maria Delia Contri, Roberto Cavasola, Antonio Di Ciaccia, Torino, Einaudi, 1994, p. 68. 
non è lo Stato, né la Legge, né la Natura: per Pentesilea la Madre è Liebe. Ella disobbedisce infatti a tutti i suoi doveri di regina, di capo e di guerriera. Disobbedisce preterintenzionalmente e dunque realmente, lasciandosi incantare dal desiderio, dal suo Oggetto del desiderio, che ella non sa cosa sia perché al di là (jenseits) di lei è spinta da una perdita oggettuale originaria, l'Amore originario, das Ding. La rivolta sta tutta in questa fedeltà alla Cosa e al lutto, anche a prezzo dell'orrore, anche a prezzo dello sparagmos. Anzi, l'orrore dello sparagmos sarà la prova della fedeltà di Pentesilea al proprio lutto, e, dunque, infine, a se stessa. Che importa se, poi, ci si perde? $\grave{E}$ un atto di libertà. Come non ricordare Antigone? Non le somiglia, Pentesilea, nel senso ultimo?

Potremmo anche parlare di sete di Verità. Non consiste forse in ciò l'essenza della ribellione kleistiana?

Abstract: Questo saggio si interroga sullo statuto di Achille nella Pentesilea di Heinrich von Kleist. L'ipotesi avanzata è che Achille incarni l'oggetto del desiderio della protagonista: con la parola "oggetto" si intende la rappresentazione tutta interiore costruita da Pentesilea intorno alla "cosa" immaginaria da cui è involontariamente trasportata. Questa "cosa" prende le sembianze femminili di un tenero e inerme ragazzo incoronato di rose, un "Achille delle rose", per così dire - dove la parola "rosa" si fa anagramma del desiderio ed emblema del sesso femminile. Nel corso dell'analisi, si rivela che il miraggio oggettuale di Pentesilea diventa quindi la proiezione della "cosa stessa", das Ding, ovvero l'amore materno, il quale, risultando per sempre perduto, si trasforma in fantasma persecutorio. Tale ipotesi si radica, prima che nel paradigma freudiano-lacaniano del desiderio, nel contesto politico e storico in cui la tragedia della regina delle Amazzoni fu ideata nonché nella filosofia trascendentale (kantiana) e idealistica (hegeliana) del soggetto.

The hypothesis I advance in this article is that the character of Achilles in Heinrich von Kleist's Penthesilea embodies the Object of desire of the female protagonist, the queen of the Amazons. By the word "Object", I mean the interiorised and imaginary representation Penthesilea phantasises within herself about an otherwise unknown "thing" desired which finally takes the feminine features of a tender and harmless boy wearing crowns and garlands of roses, an "Achilles among the roses", as it were - the word "rose" being both the anagram of "eros" and the emblem of the female sex. In the course of the analysis, I argue that this "mirage" of Penthesilea, the "thing desired", is the projection of the "Thing itself", maternal love, which, being unredeemedly lost to her, turns into a persecutory ghost. My understanding of von Kleist's Penthesilea is not rooted in Freudian and Lacanian psychoanalysis, but both in the historical and political context in which the tragedy of the queen of the Amazon was conceived and in the transcendental and idealistic philosophy of the Subject.

Keywords: von Kleist, oggetto, cosa, desiderio, materno, Pentesilea, Achille, idealismo; von Kleist, Penthesilea, Achilles, transcendentalism, idealism, object, desire, mother. 
Biodata: Massimo Stella è attualmente ricercatore in Critica Letteraria e Letterature comparate all'Università Ca' Foscari di Venezia. Dal 2015 al 2019 è stato ricercatore alla Scuola Normale Superiore di Pisa dove ha insegnato Letterature comparate per il corso dottorale in Letterature e filologie moderne e in Letteratura, Arte e Storia medievali e moderne. I suoi interessi di ricerca principali sono: la drammaturgia comparata (teatro elisabettiano-giacomiano e teatro antico greco e romano), la ricezione della tradizione classica nel modernismo europeo (inglese, francese, tedesco e portoghese), i modelli teorici di interpretazione del testo letterario tra linguistica, analisi strutturale e metodologia freudiana e lacaniana (massimo.stella@unive.it).

Massimo Stella teaches Comparative Literature and Theory of Literature at the Ca' Foscari University of Venice. His main research interests are: comparative theatre studies (Elizabethan and Jacobean drama, Ancient Greek and Roman Drama), the reception of Classical tradition in European (English, French, German, Portuguese) Modernism, theoretical analysis of the literary text at the interplay of linguistics, freudian, lacanian and structuralist methodology (massimo.stella@unive.it). 